\title{
Combining Isotopic Tracer Techniques to Increase Efficiency of Clinical Pharmacokinetic Trials in Oncology
}

\author{
Jeroen Roosendaal ${ }^{1,2} \cdot$ Hilde Rosing $^{1} \cdot$ Jos H. Beijnen ${ }^{1,2,3}$
}

Published online: 16 April 2020

(c) The Author(s) 2020

\begin{abstract}
With increasing numbers of drugs tested in oncology for smaller patient populations, fewer patients are available to answer important clinical pharmacological questions in the timeframe of clinical drug development. The quality and efficiency of trials to assess the pharmacokinetics of new drugs can be improved by making better use of available resources. One approach to do this is by making more effective use of isotopic tracer techniques. With increasing sensitivity of liquid chromatographytandem mass spectrometry analyzing equipment over the years, it has now become possible to generate much more rich, high-quality pharmacokinetic data than before. In particular we want to make a plea here for a hybrid trial approach, where both radiolabeled drug and stable isotopically labeled drug are administered to patients to assess both the absolute bioavailability and absorption, distribution, metabolism and excretion in a single clinical trial experiment.
\end{abstract}

\section{Key Points}

The quality and efficiency of trials to assess the pharmacokinetics of new drugs in oncology can be improved by making better use of available resources.

One approach to do this is by making more effective use of isotopic tracer techniques in combination with highsensitivity liquid chromatography-tandem mass spectrometry analyzing equipment.

In this article, we advocate that absolute bioavailability trials and mass balance trials could and should more often be combined into a single pharmacokinetic trial using a combined radiolabeled and stable isotopically labeled drug approach.

Jeroen Roosendaal

j.roosendaal@nki.nl

1 Department of Pharmacy and Pharmacology, Netherlands Cancer Institute-Antoni van Leeuwenhoek, Amsterdam, The Netherlands

2 Division of Pharmacoepidemiology and Clinical Pharmacology, Science Faculty, Utrecht Institute for Pharmaceutical Sciences, Utrecht University, Utrecht, The Netherlands

3 Division of Pharmacology, Netherlands Cancer Institute-Antoni Van Leeuwenhoek, Amsterdam, The Netherlands

\section{Introduction}

With the emergence of targeted therapies in oncology, fewer patients are available to answer important clinical pharmacological questions in the timeframe of clinical drug development. There has been an increase in the number of drugs developed for smaller patient populations due to a focus on molecular niche tumors and narrower stratification of subpopulations in more common tumor types [1]. For this reason, it has become increasingly important to develop innovative clinical trial designs whereby available resources are most optimally used. A lot of interest goes out to the optimization of pharmacodynamic studies, whereas there are equally good, and in our view often neglected, opportunities to improve the design and output from clinical pharmacokinetic trials.

Pharmacokinetic studies aim to elucidate the relation between dose, plasma concentrations, and therapeutic or toxic effects, and are necessary in order to employ new drugs under the best conditions of efficacy and safety [2]. Pharmacokinetic trials to investigate the absorption, distribution, metabolism, and excretion (ADME) and absolute bioavailability (ABA) are core studies in clinical drug development, and results of these trials are increasingly requested by regulatory agencies before drug registration [3, 4]. The overall impact of ABA trials in oncological drug development has increased, as most drugs that are currently in development or have recently been approved are destined for oral ingestion [5]. Although in some cases clinical pharmacokinetic 
trials can be performed in healthy volunteers, more clinically relevant data can be obtained using cancer patients instead. The increase in the number of drugs tested clearly requires a critical look at the way clinical pharmacokinetic trials are conducted.

\section{Conventional Absorption, Distribution, Metabolism, and Excretion (ADME) Trial Design}

ADME studies are most often performed using radiolabeled drug, and are, in this way, also known as mass balance trials. In this trial, the drug of interest is labeled with a radioactive tracer, in most cases ${ }^{14} \mathrm{C}$, to be able to trace all drug-related material throughout the body [6]. A ${ }^{14} \mathrm{C}-\mathrm{ADME}$ study primarily aims to determine the excretory pathways of radiolabeled drug, as well as to identify circulatory and excretory metabolites. The trial is usually conducted in approximately six to eight subjects using the recommended clinical dose and route of administration [6]. Although the administration of radioactivity to patients or healthy volunteers is in general not desired, an effective dose limit of $30 \mathrm{mSv}$ is considered safe for investigational purposes, keeping the 'as low as reasonably achievable' (ALARA) principle in mind [6]. As it is not always possible to calculate the effective dose prior to a ${ }^{14} \mathrm{C}$-ADME trial, a radioactive dose limit of $100 \mu \mathrm{Ci}(3.7 \mathrm{MBq})$ is often used in practice and is also considered safe [6] ${ }^{14} \mathrm{C}$-ADME trials are performed for virtually all small molecule drugs before market approval, as they are considered essential to allow for safe and effective drug use in the clinic, and are strongly recommended for anticancer drug development by regulatory agencies $[7,8]$.

\section{Conventional Absolute Bioavailability (ABA) Trial Design}

ABA studies are traditionally performed by administering an extravascular and intravenous dose of drug in a crossover design with a washout period in between [9]. On one dosing occasion, the drug is administered by one route (for example, orally), and plasma samples are taken at appropriate times and analyzed for parent drug. Following a suitable washout period, the same subjects are administered the drug intravenously and, again, plasma samples are taken over time and analyzed for parent drug. The total area under the plasma concentration-time curve (AUC) is calculated for each dose route using Eq. 1, where $\mathrm{CL}$ is the clearance of the drug and $F$ is the bioavailability.

$F \times$ Dose $=\mathrm{CL} \times$ AUC

For intravenous administration, $100 \%$ of the drug is taken to enter the systemic circulation and therefore $F$ per definition equals 1 . For extravascular administration, only a proportion of the dose might be absorbed systemically intact, reflected by a change in $F$. Dividing the equations for intravenous (iv) and extravascular (ev) administration gives the classical equation for calculating bioavailability based on plasma data, as shown in Eq. 2, based on the assumption of linear pharmacokinetics and an equal clearance for both the extravascular and intravenous gift.

$F=\left(\frac{\mathrm{AUC}_{\mathrm{ev}}}{\mathrm{AUC}_{\mathrm{iv}}}\right) \times\left(\frac{\text { Dose }_{\mathrm{iv}}}{\text { Dose }_{\mathrm{ev}}}\right)$

Although recommended by regulatory agencies, data on the ABA are not always presented at the time of drug approval. For the group of tyrosine kinase inhibitors, an important class of oral targeted therapies in oncology, data on the ABA have not been reported for 10 of 20 of the drugs registered by both the EMA and US FDA to 2014 [5]. The main reason for this is thought to be the poor solubility of these compounds, making it challenging to develop a therapeutic intravenous solution for clinical evaluation, which is required to perform an $\mathrm{ABA}$ trial using the conventional crossover design. A potential way to overcome this problem is by making use of a microdosing trial design [9].

\section{ABA Microdosing Trials}

Microdosing trials can be used for the exploration and investigation of many pharmacokinetic properties, including the assessment of ABA. A microdose is defined as 1/100th of the therapeutic dose, with a maximum of $100 \mu \mathrm{g}$. By making use of an intravenous microdose instead of an intravenous therapeutic dose, solubility issues can be circumvented, as only a maximum of $100 \mu \mathrm{g}$ of drug needs to be dissolved in an intravenous formulation. Furthermore, additional safety testing of microdose formulations is not required per FDA guidelines because of the low dose [10]. In an ABA microdosing trial, the oral therapeutic dose intended for clinical use is administered, after which the intravenous microdose is administered at the estimated maximum plasma concentration $\left(C_{\max }\right)$ of the oral gift. In this manner, the ABA can then be calculated the same way as for the conventional trial design (Eq. 2). This approach was first introduced in 2006, where the ABA of three non-oncolytic drugs was successfully assessed following simultaneous administration of a $100 \mu \mathrm{g}{ }^{14} \mathrm{C}$-labeled microdose with an oral therapeutic dose [11].

A major advantage of using the microdose approach for ABA determination is that the intravenous microdose can be administered to a patient during the same dose event as the extravascular gift. To be able to use this trial design, there is however a need to differentiate between the intravenous 
and extravascular drug exposure to be able to calculate the ABA. This differentiation can be achieved by using drug labels incorporated into the drug of interest for intravenous administration, using either ${ }^{14} \mathrm{C}$-radiolabels or stable isotope labels (e.g. ${ }^{13} \mathrm{C},{ }^{15} \mathrm{~N}$ ). Depending on the label of choice, different analytical techniques are required to quantify the very low exposure to microdosed labeled drug in the systemic circulation. For ${ }^{14} \mathrm{C}$-radiolabeled drug, accelerator mass spectrometry (AMS) is applied, where the ratio of carbon atoms $\left({ }^{14} \mathrm{C}:{ }^{12} \mathrm{C}\right)$ is analyzed, independent of the molecular mass or structure of the drug. For stable isotopically labeled drug, liquid chromatography coupled with tandem mass spectrometry (LC-MS/MS) is used, where the analysis is based on the mass difference between labeled and unlabeled drug following incorporation of heavier stable isotopes into the drug of interest. Use of the microdose trial design results in less dose events required and a more accurate calculation of ABA, as all measurements take place in a single subject during a single-dose event, eliminating intrasubject variability and concentration-dependent clearance.

Initially, microdose trials were performed exclusively using a combination of AMS and ${ }^{14} \mathrm{C}$-labeled drug, due to the superior sensitivity of this approach (up to the attomole range). However, a major drawback of AMS is that it is very time-consuming, costly, and analytically challenging, limiting its general practical usability [12]. Furthermore, the calculation of the exposure to unlabeled drug still requires an LC-MS/MS method next to an AMS method, complicating sample processing and analysis and prolonging study duration. At the moment, AMS technologies are advancing to overcome some of these problems by the development of compact AMS systems with automated sample handling and a direct coupling with liquid chromatography systems. In addition, simpler laser-based techniques for ${ }^{14} \mathrm{C}$-detection are being developed that might replace AMS as a core analytical technology in the future [13]. Despite these developments, a drawback inherent to the use of ${ }^{14} \mathrm{C}$ as an isotopic tracer is that the use of a ${ }^{14} \mathrm{C}$-microdose for $\mathrm{ABA}$ determination cannot be combined with ${ }^{14} \mathrm{C}$-ADME trials, as it is impossible to distinguish the intravenous ${ }^{14} \mathrm{C}$-microdose for ABA calculation from the oral ${ }^{14} \mathrm{C}$-dose for ADME characterization in biological samples, when both are administered at the same time.

Recent developments in the field of mass spectrometry paved the way to use LC-MS/MS as an alternative for AMS to perform ABA microdose trials [14-17]. Triple quadrupole mass spectrometers are one of the most commonly sold types of LC-MS/MS and are considered superior in sensitivity for LC-MS/MS-based drug quantification [18]. LC-MS/ MS assays using the triple quadrupole principle now report lower limits of quantification in the $\mathrm{pg} / \mathrm{mL}$ range, reaching sensitivity levels that were previously only possible for AMS [19]. Although the sensitivity of AMS is still far superior to LC-MS/MS (as low as $10^{-18} \mathrm{~g} / \mathrm{mL}$ for AMS vs. $10^{-12} \mathrm{~g} /$ $\mathrm{mL}$ for LC-MS/MS [19]), it is not always required to reach this level of sensitivity for the purpose of a clinical microdose trial. Recently, we have successfully demonstrated the application of an LC-MS/MS method for the support of a microdose trial with gemcitabine, with a detection limit in the low $\mathrm{pg} / \mathrm{mL}$ range [20].

\section{Combining ${ }^{14} \mathrm{C}$-ADME and ABA Microdosing Trials}

For most drugs, ${ }^{14} \mathrm{C}$-ADME trials and $\mathrm{ABA}$ trials are performed separately using conventional trial designs, as depicted in Fig. 1a. For a ${ }^{14} \mathrm{C}$-ADME trial, a ${ }^{14} \mathrm{C}$-radiolabeled dose possibly mixed with unlabeled $\left({ }^{12} \mathrm{C}\right)$ drug is administered to a study subject orally, after which samples are collected until most of the administered radioactivity (usually $>80 \%$ [6]) is recovered in excreta (urine, feces). During this time period, part of the investigations comprise the collection of blood samples for the quantification of total ${ }^{14} \mathrm{C}$-radioactivity and unlabeled $\left({ }^{12} \mathrm{C}\right)$ drug in plasma using liquid scintillation counting (LSC) and LC-MS/MS, respectively.

For an ABA trial, subjects receive both an intravenous and oral unlabeled dose at therapeutic strength during separate dosing occasions, with a washout period in between. This will result in two separate dose events and sample collection periods (Fig. 1a). For each dose event, the plasma exposure to unlabeled drug is calculated following LC-MS/MS analysis, after which the ABA of the oral drug formulation can be determined.

Assuming the inclusion of six subjects per trial (based on the number most often used for ${ }^{14} \mathrm{C}$-ADME trials [6]), when both studies are conducted separately during clinical drug development, a total of at least 12 subjects receiving a total of three dose events (Fig. 1) are required to be able to investigate both the ABA and ADME characteristics of a new drug. These procedures can be simplified by making use of a hybrid trial design.

\subsection{Hybrid Trial Study Design}

Using the hybrid trial design, an oral dose of ${ }^{14} \mathrm{C}$-radiolabeled drug mixed with unlabeled $\left({ }^{12} \mathrm{C}\right)$ drug and an intravenous microdose of stable isotopically labeled drug are administered to a study subject to determine both the 
A

${ }^{14}$ C-ADME trial

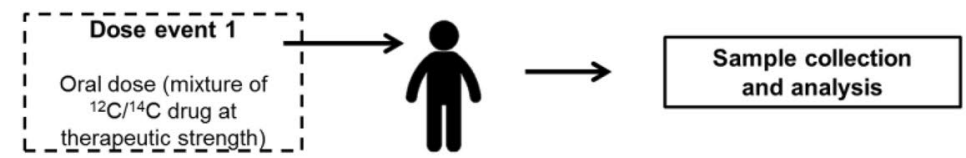

\section{Conventional two-period crossover ABA trial}

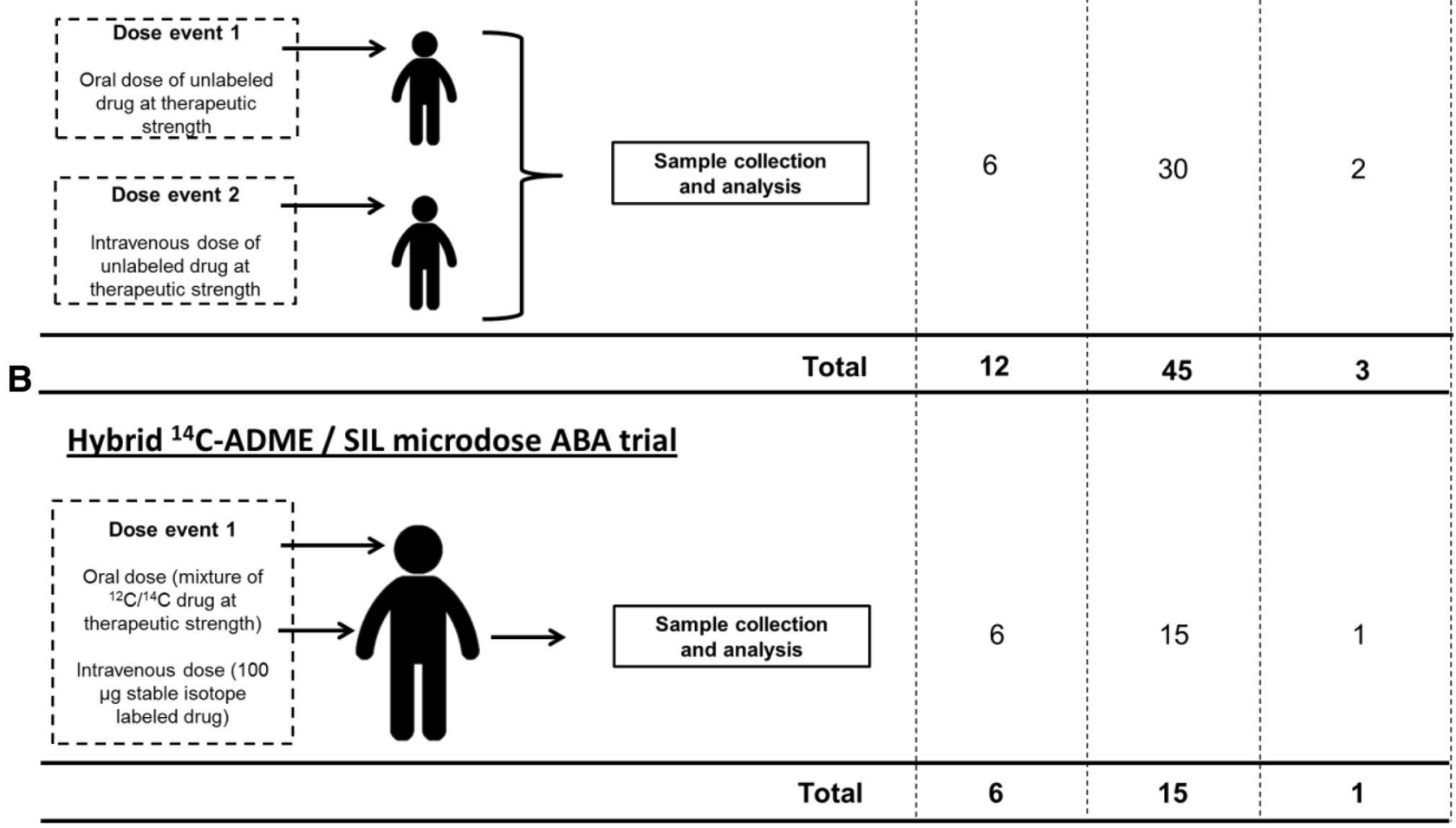

Fig. 1 Schematic overview of number of subjects, samples, and dose events required for the execution of a a ${ }^{14} \mathrm{C}-\mathrm{ADME}$ trial and a separate conventional two-period crossover ABA trial; and $\mathbf{b}$ a ${ }^{14} \mathrm{C}$-ADME trial combined with an SIL microdose ABA trial. A theoretical number of 6 subjects for $\mathrm{ABA}$ and ${ }^{14} \mathrm{C}$-ADME determinations

$\mathrm{ABA}$ and $\mathrm{ADME}$ characteristics during a single-dose event (Fig. 1b). After administration of an oral ${ }^{14} \mathrm{C} /{ }^{12} \mathrm{C}$ dose, an intravenous stable isotopically labeled microdose is administered at the estimated $\mathrm{C}_{\max }$ of orally administered drug. Plasma samples are collected for the analysis of total ${ }^{14} \mathrm{C}$-radioactivity using LSC, and for the simultaneous analysis of stable isotopically labeled and unlabeled drug using LC-MS/MS. The plasma sample collection period for the hybrid trial design is equal to the collection period using a conventional ${ }^{14} \mathrm{C}$-ADME trial, and is based on the criteria for recovery of radioactivity in excreta [6]. was used, as well as 15 samples for concentration-time curve establishment per dose event. $A B A$ absolute bioavailability, $A D M E$ absorption, distribution, metabolism, and excretion, SIL stable isotopically labeled

\subsection{Advantages of Using a Hybrid Trial Approach}

The principal advantage of using a hybrid trial approach is that it requires less subjects, dose events, and samples to determine the $\mathrm{ABA}$ and $\mathrm{ADME}$ characteristics of a new drug. Using conventional trial designs, multiple-dose events result in the need to collect and analyze samples for total ${ }^{14} \mathrm{C}$-radioactivity and unlabeled drug (Fig. 2a), as well as exposure after intravenous and oral administration of a therapeutic dose (Fig. 2b), separately. Using the hybrid trial design, plasma concentration-time curves for total 
${ }^{14} \mathrm{C}$-radioactivity, unlabeled drug, and stable isotopically labeled microdose can be generated following sample collection after a single-dose event, as depicted in Fig. 2c.

The intravenously administered stable isotopically labeled microdose can be distinguished from orally administered ${ }^{14} \mathrm{C} /{ }^{12} \mathrm{C}$ drug by virtue of their different molecular masses, using simultaneous LC-MS/MS detection. This way, the ABA of the orally administered drug can be calculated by dividing oral exposure to unlabeled drug by the dose-normalized intravenous exposure to stable isotopically labeled drug in plasma. The principal advantage of the isotopic method is that the plasma drug concentration relating to the intravenous and oral doses are measured in the same plasma samples, thereby eliminating interoccasional variability and concentration-dependent clearance.

For ADME investigations, the total amount of radioactivity in the circulation can be analyzed using LSC, in the same way as for a conventional ${ }^{14} \mathrm{C}$-ADME trial. As the total amount of ${ }^{14} \mathrm{C}$-radioactivity in the body comprises parent drug and metabolites bearing the radioactive label, the total amount of radioactivity in plasma is always equal to or higher than the concentration of parent drug, as illustrated in Fig. 2a. This ensures that the sample collection period required to determine the ADME characteristics of a drug is always sufficient to determine the ABA following an intravenous microdose administration. The combined assessment of $\mathrm{ABA}$ and $\mathrm{ADME}$ characteristics during a single-dose event allows for the direct determination of key pharmacokinetic parameters, such as clearance, volume of distribution and fraction of drug metabolized after oral administration [21]. This results in an increased amount of high-quality data, generated using the same amount of resources and time required to conduct a conventional ${ }^{14} \mathrm{C}-\mathrm{ADME}$ trial.

\subsection{Requirements to Perform a Hybrid Trial}

There are three main requirements to assess if a hybrid trial approach is feasible. First, information on a limited number of pharmacokinetic parameters of the drug of interest is needed to be able to calculate the expected plasma drug exposure after microdose administration. These parameters are often available after a first-in-human (FIH) clinical trial. Second, a sensitive and selective LC-MS/MS method is required to be able to detect low concentrations of stable isotopically labeled drug in the presence of high concentrations of unlabeled drug. Third, stable isotopically labeled drug and ${ }^{14} \mathrm{C}$-radiolabeled drug need to be synthesized to allow for the development of labeled drug formulations.

At the start of clinical drug development, phase I FIH trials often provide data on the $C_{\max }$, apparent volume of distribution after extravascular administration $\left(V_{\mathrm{d}} / F\right)$, and elimination half-life $\left(t_{1 / 2}\right)$ of the drug of interest after administration of a known extravascular dose at the intended therapeutic concentration. Using these parameters, the theoretical $C_{\max }$ and minimum plasma concentration $\left(C_{\min }\right)$ up to a certain time point following a $100 \mu \mathrm{g}$ intravenous microdose can be estimated. These concentrations can then be used to determine the required sensitivity and concentration range of the LC-MS/MS method. Assume a microdose is administered intravenously at a dose of $100 \mu \mathrm{g}$, with a $V_{\mathrm{d}} / F$ of $1000 \mathrm{~L}$, the expected $C_{\max }$ will be $100 \mathrm{pg} / \mathrm{mL}$, with a $C_{\min }$ of $10 \mathrm{pg} / \mathrm{mL}$ after a little more than three half-lives. In this case, an LLOQ of at least $10 \mathrm{pg} / \mathrm{mL}$ is required for a reliable measurement of the AUC from time zero extrapolated to infinity $\left(\mathrm{AUC}_{\infty}\right)$. If the dose decreases or the $V_{\mathrm{d}} / F$ increases, demands on the assay sensitivity become increasingly intense.

As both unlabeled and stable isotopically labeled drug are present in the circulation at the same time, there is a need to selectively quantify both compounds. The number of labels required to distinguish the intravenous microdose from the unlabeled extravascular dose can easily be determined using isotopic distribution pattern software and the method described by $\mathrm{Gu}$ et al. [22, 23], in combination with the known $C_{\max }$ of the extravascular dose and the expected $C_{\max }$ of the intravenous microdose. When using an intravenously administered stable isotopically labeled microdose, there is potential that the presence of the isotope alters reaction rates in vivo because the atomic bond strength is dependent on the mass of the bonded isotope. This is known as the kinetic isotope effect (KIE), and the magnitude of this effect is dependent on the difference in mass of the bonded isotopes, as well as the location of the isotopic labels in the drug molecule. The KIE can be significant with lower-mass isotopes, such as deuterium, but is virtually insignificant with isotopes of carbon and nitrogen [24]. The use of carbon or nitrogen labels for stable isotopically labeled microdose formulations is therefore preferred. Next to stable isotopically labeled drug for intravenous microdose administration, an alternative internal standard is required for the LC-MS/MS assay to allow for simultaneous quantification of both orally and intravenously administered drug. The number of labels required for the internal standard can also be calculated using isotope distribution software [23], and is normally less than the number of labels required for the stable isotopically labeled microdose. The internal standard concentration used is dependent on the concentrations of unlabeled and labeled drug in plasma and should result in accurate quantification of both unlabeled and labeled drug, without causing crossanalyte interference $[7,25]$.

If technologically feasible, all that is required next is to synthesize radiolabeled and stable isotopically labeled drug, which may already (partially) be done for preclinical investigations, and to develop an intravenous microdose formulation, which most often can be very straightforward 

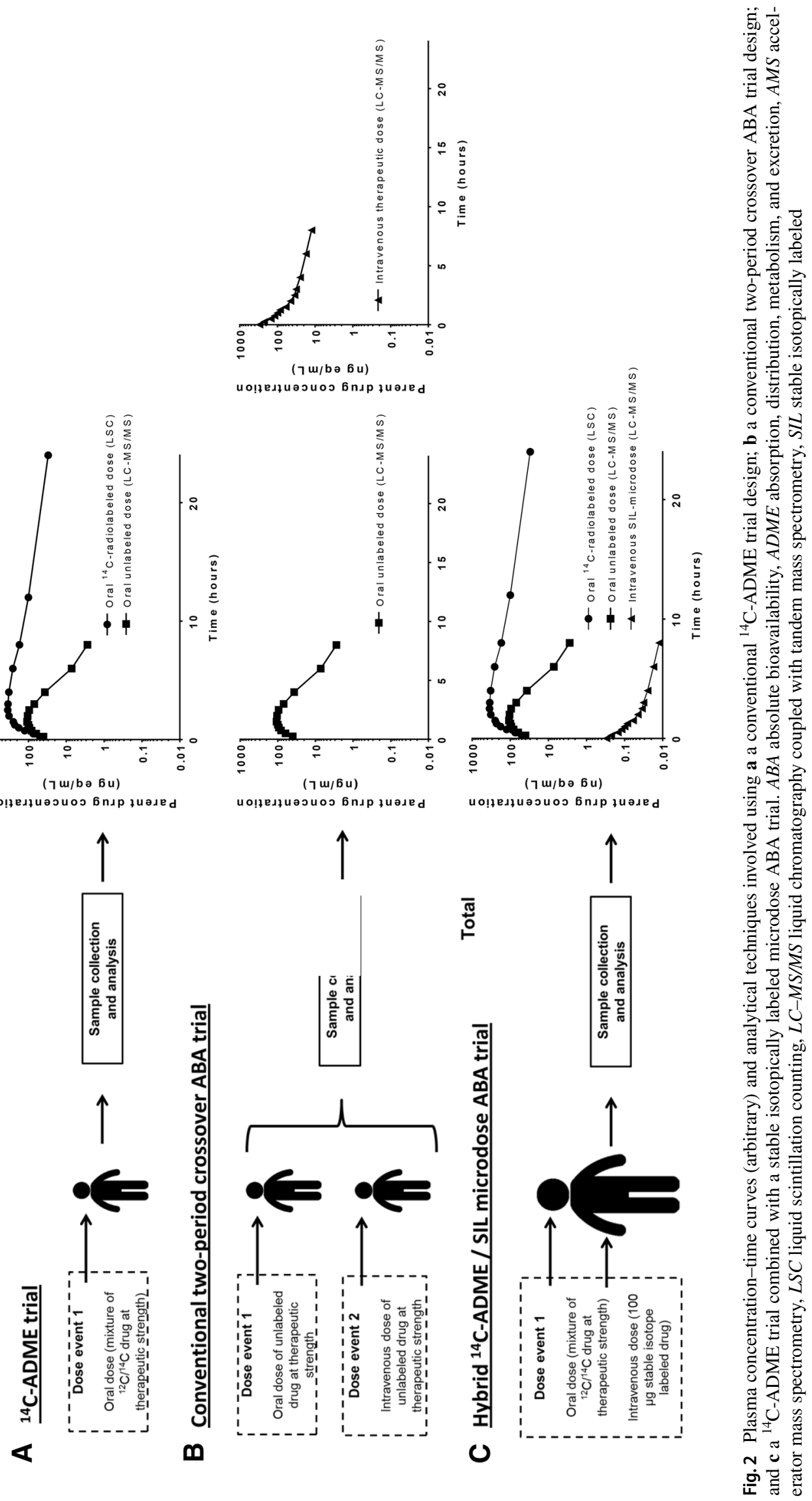
because of the low amount of drug that needs to be dissolved in aqueous media [9].

\subsection{Examples of Using a Hybrid Trial Approach}

Despite the technological advances and opportunities that currently exist, $\mathrm{ABA}$ and ${ }^{14} \mathrm{C}$-ADME pharmacokinetic trials are still most often performed separately. The feasibility of using a double-tracer approach in a single clinical trial, combining stable isotopically labeled drug with radiolabeled drug, has already successfully been demonstrated for two drugs outside oncology [21, 26]. For tofogliflozin, a drug used for the treatment of type 2 diabetes mellitus, ${ }^{14} \mathrm{C}$-radiolabeled tofogliflozin was orally administered to patients at the same time as an intravenous ${ }^{13} \mathrm{C}$-stable isotopically labeled tofogliflozin microdose [21]. For basimglurant, a drug under clinical development for major depressive disorder, the same approach was followed, whereby ${ }^{14} \mathrm{C}$-radiolabeled basimglurant was orally administered to patients at the same time as an intravenous ${ }^{13} \mathrm{C}_{6}$-stable isotopically labeled basimglurant microdose [26]. For both drugs, the double-tracer approach allowed for the simultaneous characterization of both the ABA and ADME characteristics of these drugs in a single study, during a single-dose event.

Thus far, to our current knowledge, a double-tracer approach has been described for these two drugs only, and has not yet been performed in oncological drug development. This raises the question as to what causes a further lack of implementation of this promising and efficient approach. It can be argued that the timing of ABA and ${ }^{14} \mathrm{C}$-ADME trials during clinical drug development is different. The assessment of ABA ideally takes place as early as possible, e.g. to allow for adjustment of the pharmaceutical formulation based on study outcome. ${ }^{14} \mathrm{C}$-ADME trials take place from early (phase I) to late (phase III) clinical drug development, ideally after the recommended phase II dose has been established [6]. However, in practice, it can occur that the ABA and ${ }^{14} \mathrm{C}$-ADME trials are conducted around the same time, allowing for combined trial conduction. What has also been observed is that thus far, the use of a stable isotopically labeled microdose to assess the ABA has been described for one oncological drug only hitherto [27]. ABA microdose trials are still most often performed using intravenously administered ${ }^{14} \mathrm{C}$-labeled drug [24], making it impossible to perform a ${ }^{14} \mathrm{C}$-ADME trial during the same dose event, as there is no way to differentiate between the intravenous and oral dose when using the same drug label. A transition in using stable isotopically labeled microdoses instead of ${ }^{14} \mathrm{C}$-labeled microdoses for the assessment of ABA may result in increased potential for using the hybrid trial approach that we propose. $\mathrm{As}{ }^{14} \mathrm{C}$-ADME trials are performed for virtually all small molecule drugs in clinical development, it would take little effort to also add ABA assessment, using a stable isotopically labeled microdose, to this trial.

\section{Conclusions}

We advocate that for novel extravascular anticancer agents evaluated in oncology, ABA and ${ }^{14} \mathrm{C}$-ADME trials should be combined into a single pharmacokinetic trial using a combined radiolabeled and stable isotopically labeled drug approach. This double-tracer approach has several advantages over the traditional approach, where ABA and ${ }^{14} \mathrm{C}$-ADME trials are performed separately. A double-tracer approach results in less required subjects, less samples, less dose events, lower costs, less time needed for clinical drug development, and results that better reflect use of the drug in clinical practice. The double-tracer trial is therefore an elegant way to obtain effective and efficient pivotal clinical pharmacokinetic data during clinical drug development.

\section{Compliance with Ethical Standards}

Conflicts of Interest Jeroen Roosendaal, Hilde Rosing, and Jos Beijnen declare no potential conflicts of interest with respect to the research, authorship, and/or publication of this article.

Funding The authors received no financial support for the research, authorship, and/or publication of this article.

Open Access This article is licensed under a Creative Commons Attribution-NonCommercial 4.0 International License, which permits any non-commercial use, sharing, adaptation, distribution and reproduction in any medium or format, as long as you give appropriate credit to the original author(s) and the source, provide a link to the Creative Commons licence, and indicate if changes were made. The images or other third party material in this article are included in the article's Creative Commons licence, unless indicated otherwise in a credit line to the material. If material is not included in the article's Creative Commons licence and your intended use is not permitted by statutory regulation or exceeds the permitted use, you will need to obtain permission directly from the copyright holder.To view a copy of this licence, visit http://creativecommons.org/licenses/by-nc/4.0/.

\section{References}

1. Verweij J, Hendriks HR, Zwierzina H. Innovation in oncology clinical trial design. Cancer Treat Rev. 2019;74:15-20.

2. European Medicines Agency. Pharmacokinetic studies in man. 1987. https://www.ema.europa.eu/en/documents/scientific-guide line/pharmacokinetic-studies-man_en.pdf. Accessed 25 May 2019

3. European Medicines Agency. Clinical pharmacology and pharmacokinetics: questions and answers. 2019. https://www.ema.europ a.eu/en/human-regulatory/research-development/scientific-guide lines/clinical-pharmacology-pharmacokinetics/clinical-pharm acology-pharmacokinetics-questions-answers. Accessed 26 May 2019 
4. US FDA. Bioavailability studies submitted in NDAs or INDsgeneral considerations and guidance for industry. Silver Spring, MD: US FDA; 2019.

5. Herbrink M, Nuijen B, Schellens JHM, Beijnen JH. Variability in bioavailability of small molecular tyrosine kinase inhibitors. Cancer Treat Rev. 2015;41:412-22.

6. Nijenhuis CM, Schellens JHM, Beijnen JH. Regulatory aspects of human radiolabeled mass balance studies in oncology: concise review. Drug Metab Rev. 2016;48:266-80.

7. European Medicines Agency. Guideline on the evaluation of anticancer medicinal products in man. Amsterdam: European Medicines Agency; 2017.

8. US FDA. Guidance for industry - safety testing of drug metabolites. Silver Spring, MD: US FDA; 2016

9. Lappin G, Rowland M, Garner RC. The use of isotopes in the determination of absolute bioavailability of drugs in humans. Expert Opin Drug Metab Toxicol. 2006;2:419-27.

10. US FDA. Exploratory IND Studies. Silver Spring, MD: US FDA; 2006.

11. Lappin G, Kuhnz W, Jochemsen R, Kneer J, Chaudhary A, Oosterhuis $\mathrm{B}$, et al. Use of microdosing to predict pharmacokinetics at the therapeutic dose: experience with 5 drugs. Clin Pharmacol Ther. 2006;80:203-15.

12. Garner RC. Practical experience of using human microdosing with AMS analysis to obtain early human drug metabolism and PK data. Bioanalysis. 2010;2:429-40.

13. Vuong LT, Song Q, Lee HJ, Roffel AF, Shin S-H, Shin YG, et al. Opportunities in low-level radiocarbon microtracing: applications and new technology. Futur Sci OA. 2015;2:FSO74.

14. Ings RM. Microdosing: a valuable tool for accelerating drug development and the role of bioanalytical methods in meeting the challenge. Bioanalysis. 2009;1:1293-305.

15. Lappin G, Wagner CC, Langer O, Van de Merbel N. New ultrasensitive detection technologies and techniques for use in microdosing studies. Bioanalysis. 2009;1:357-66.

16. Lappin G, Noveck R, Burt T. Microdosing and drug development: past, present and future. Expert Opin Drug Metab Toxicol. 2013;9:817-34.

17. Maeda K, Sugiyama Y. Novel strategies for microdose studies using non-radiolabeled compounds. Adv Drug Deliv Rev. 2011;63:532-8.

18. Haag AM. Mass analyzers and mass spectrometers. Adv Exp Med Biol. 2016;919:157-69.
19. Burt T, John CS, Ruckle JL, Vuong LT. Phase-0/microdosing studies using PET, AMS, and LC-MS/MS: a range of study methodologies and conduct considerations. Accelerating development of novel pharmaceuticals through safe testing in humans-a practical guide. Expert Opin Drug Deliv. 2017;14:657-72.

20. Van Nuland M, Hillebrand MJX, Rosing H, Burgers JA, Schellens JHM, Beijnen JH. Ultra-sensitive LC-MS/MS method for the quantification of gemcitabine and its metabolite $2^{\prime}, 2^{\prime}$-difluorodeoxyuridine in human plasma for a microdose clinical trial. J Pharm Biomed Anal. 2018;151:25-31.

21. Schwab D, Portron A, Backholer Z, Lausecker B, Kawashima K. A novel double-tracer technique to characterize absorption, distribution, metabolism and excretion (ADME) of [14C]tofogliflozin after oral administration and concomitant intravenous microdose administration of [13C]tofogliflozin in humans. Clin Pharmacokinet. 2013;52:463-73.

22. Gu H, Wang J, Aubry AF, Jiang H, Zeng J, Easter J, et al. Calculation and mitigation of isotopic interferences in liquid chromatography-mass spectrometry/mass spectrometry assays and its application in supporting microdose absolute bioavailability studies. Anal Chem. 2012;84:4844-50.

23. SIS Web. 2019. Isotope distribution calculator and mass spec plotter. https://www.sisweb.com/mstools/isotope.htm. Accessed 25 May 2019.

24. Lappin G. Approaches to intravenous clinical pharmacokinetics: recent developments with isotopic microtracers. J Clin Pharmacol. 2016;56(1):11-23.

25. US FDA. Guidance for Industry: Bioanalytical Method Validation. 2018. p. 1-44. https://www.fda.gov/downloads/drugs/guidances/ ucm070107.Pdf. Accessed 8 Jul 2019.

26. Guerini E, Schadt S, Greig G, Haas R, Husser C, Zell M, et al. A double-tracer technique to characterize absorption, distribution, metabolism and excretion (ADME) of [(14)C]-basimglurant and absolute bioavailability after oral administration and concomitant intravenous microdose administration of [(13)C6]-labeled basimglurant in humans. Xenobiotica. 2017;47(2):144-53.

27. De Vries R, Smit JW, Hellemans P, Jiao J, Murphy J, Skee D, et al. Stable isotope-labelled intravenous microdose for absolute bioavailability and effect of grapefruit juice on ibrutinib in healthy adults. Br J Clin Pharmacol. 2016;81:235-45. 